\title{
Color Defect Detection of Pharmaceutical Capsules using Histogram based Thresholding
}

\author{
Hardeep Kaur*, Kamaldeep and Garima \\ ECE Department, Lovely Professional University, Phagwara - 144411, Punjab, India; \\ hardeep.17208@|pu.co.in, kamaldeep.16958@lpu.co.in, garima.17818@lpu.co.in
}

\begin{abstract}
In this paper, a new method of inspecting pharmaceutical capsules aesthetic defects is proposed using color image processing. After an image is taken, median filtering is performed. Then the region based statistics is calculated to acquire the largest area of pharmaceutical capsule from the image. After that RGB image is transferred into R plane, G plane and B plane. Then histogram of each plane is calculated. Further by setting thresholding value of each plane, mask of each plane is determined. Color segmentation of image is achieved by combining mask of each plane. Experiments are conducted with the quality inspection of pharmaceutical capsules. Time is also taken as major parameter of the system. Experiment results show the reliability and feasibility of proposed method as time and quality control are two main parameters in industry. The proposed paper present an application to detect defect related to color of pharmaceutical capsules which can be adapted to other practical applications for automated visual inspection using image processing techniques.
\end{abstract}

Keywords: Color Segmentation, Histogram, Image Enhancement, RGB Planes, Thresholding

\section{Introduction}

Nowadays, pharmaceutical companies produce a vast amount of different capsules worldwide. Various defects in capsules can arise both during production and transportation process. So, visual inspection is very demanding as capsules come in different shape, size and color and have different defects.

Color ${ }^{1}$ is one of the most important features in an image and is the elementary information stored in pixels. Thresholding is usually applied for objects from background discrimination. The purpose of thresholding is to extract those pixels from some image which represent an object. Though the information is binary the pixels represent a range of intensities. In general, the grey levels of pixels that belong to the object are substantially different from the gray levels of pixels that belong to the background, then thresholding becomes straightforward and the most effective tool to extract objects from background. Histogram based thresholding $\underline{\underline{3}}$ methods have been intensively studied and are the most common methods applied. In this method, the optimal threshold of the entire image or part of image is determined according to the characteristics of the image's histogram. If the histogram is bimodal, then this method can effectively extract the foreground from background.

\section{Work Approach}

First step is image enhancement to denoise the image. Median filtering is done that replaces the value of a pixel by the median of the intensity levels in the neighborhood of that pixel. The value of pixel at $(\mathrm{x}, \mathrm{y})$ is included in the computation of the median. Median filters are quite popular because, for certain types of random noise, they provide excellent noise-reduction capabilities, with considerably less blurring than linear smoothing filters of similar size.

Second step is region based statistics ${ }^{\underline{3}}$ measure the properties of image regions. Biggest area is computed and

${ }^{*}$ Author for correspondence 
further properties of that region are calculated. One of the parameter is pixel measurement that is mean intensity is calculated. Object from the image is extracted and further processing is done on it.

Third step is color segmentation. The image is converted to RGB planes ${ }^{4}$ and histogram ${ }^{\underline{5}}$ of each plane is calculated. By setting the threshold of each plane based on intensity, mask of each plane is determined such that it represent object that fall in that range of intensity. Combining all three planes mask we get the segmented image. The proposed work approach is shown in the following flowchart Figure 1.

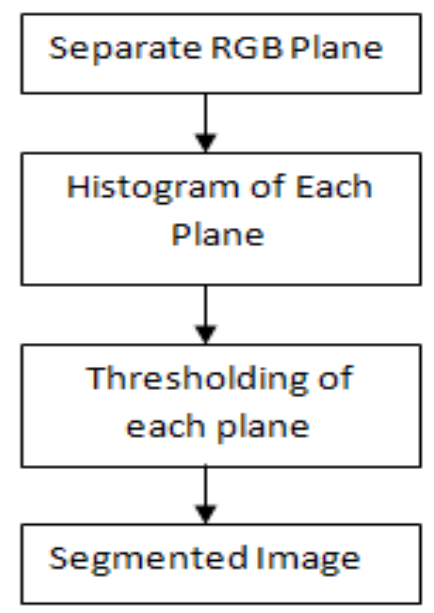

Figure 1. Flowchart of proposed work approach.

\section{Results}

Firstly, RGB images are separated into three planes that are red, blue and green planes. Further the histogram of each plane is calculated. By setting the threshold of each plane based on intensity, mask of each plane is determined such that it represent object that fall in that range of intensity. Combining all three planes mask we get the segmented image.

The proposed work approach is run over the database of images of pharmaceutical color capsules using MATLAB 7.0. Here we have taken the capsules of double color blue and white. Figure 2 shows no defect detected in pharmaceutical capsule and Figure 3 shows the detected defects.

Time taken per capsule to be examined is a very important parameter for this research work. So, the algorithm is run over number of images and time taken to execute is noted down in Table 1.

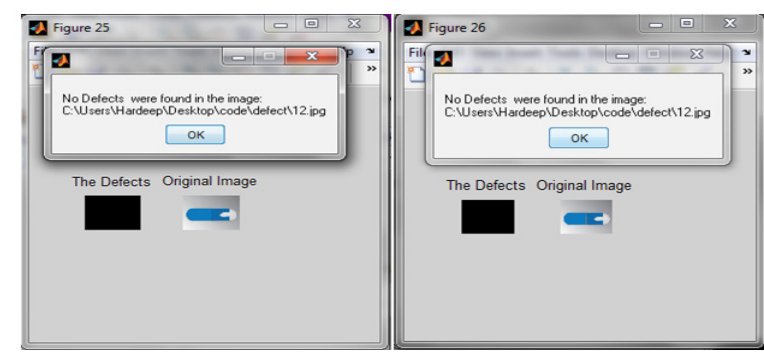

Figure 2. No defect in pharmaceutical capsule.

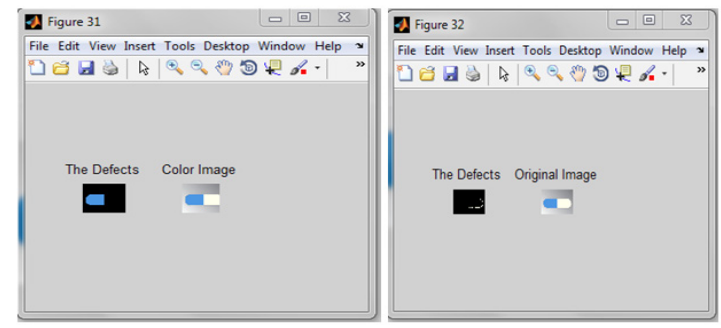

Figure 3. Color defect detection.

Table 1. Time taken to examine each capsule

\begin{tabular}{|l|l|}
\hline Type of Algorithm & $\begin{array}{l}\text { Approximate time taken to } \\
\text { examine each capsule (millisec) }\end{array}$ \\
\hline Histogram algorithm & 320 \\
\hline
\end{tabular}

\section{Conclusion}

Defect detection using image in industry is very essential. Using color segmentation, aesthetic defects that lead to customer unsatisfaction are detected. Histogram and thresholding based on intensity determine the color defects. The program is implemented using GUI is applicable to other types of industrial products and so it can be used as a general purpose real time automated visual inspection software.

\section{Future Scope}

A lot of work can be done further in this research. Firstly, the threshold value which is selected manually can be done automatic depending on the intensity. Secondly, more improvement in time taken per capsule can be chased.

\section{References}

1. Wang X, Huang X. A color texture segmentation method to extract tree image in complex scene. 2010 IEEE 
International Conference on Machine Vision and Humanmachine Interface; 2010. p. 621-5.

2. Huang S, Ahmadi M, Sid-Ahmed MA. An edge based thresholding method. 2006 IEEE International Conference on Systems, Man and Cybernetics, SMC '06; 2006 Oct.

3. Riyadi S, Abd Rahni AA, et al. Shape characteristic analysis for papaya size classification. 2007 IEEE 5th Student Conference on Research and Development; 2007 Dec.

4. Thilepa R. A paper on automatic fabrics fault processing using image processing techniques in MATLAB. An International Journal. $2010 \mathrm{Dec} ; 1$.

5. Jau UL, Siong C. A comparison of RGB and HSI color segmentation in real-time video Images: A Preliminary study on road sign detection. 2008 International Symposium on Information Technology, ITSim; 2008 Aug.
6. Wei CC, Wang CH Huang SW. Using threshold method to separate the edge, coating and body of tongue in automatic tongue diagnosis. 2010 Sixth International Conference on Networked Computing and Advanced Information Management (NCM); 2010. p. 653-6.

7. Nimbarte NM, Mushrif MM. Multi-level thresholding algorithm for color image segmentation. 2010 IEEE 2nd International Conference on Computer Engineering and Application; 2010. p. 231-3.

8. Jianjun, et al. Segmentation method of fruit image and comparative experiments. 2008 IEEE International Conference on Computer Science and Software Engineering; 2008. p. 1098-102. 